\title{
UNIQUE FACTORIZATION IN RINGS WITH RIGHT ACC
}

\author{
by H. H. BRUNGS
}

(Received 2 May, 1977)

If $R$ is an integral domain with maximum condition for principal right ideals-right $\mathrm{ACC}_{1}$-every nonzero non-unit in $R$ has irreducible factors, but is not necessarily a product of such factors. Using additional basic factors - called infinite primes in [1] results about unique factorization in principal right ideal domains have been obtained in [1], [2], and [5].

In this paper we will define generalized atoms, more exactly $\alpha$-atoms, $\alpha$ an ordinal, for any integral domain $R$ with right $\mathrm{ACC}_{1}$. The 1-atoms are just the irreducible elements. Every nonzero non-unit in $R$ can be written as a finite product of generalized atoms. If $R$ is a domain with modular factor lattice, i.e. $V(a)=\{b R: a \in b R\}$ is a modular lattice with respect to inclusion for every nonzero element $a$ in $R$, any two factorizations of a nonzero element into products of generalized atoms contain the same number of $\alpha$-atoms for a fixed ordinal $\alpha$, provided no $\beta$-atom with $\beta<\alpha$ precedes an $\alpha$-atom in these factorizations (Theorem 1). Sharper results are obtained in case $R$ is a weak Bezout domain or even a local weak Bezout domain.

Let $R$ be an integral domain with right $\mathrm{ACC}_{1}$. We write $R^{*}$ for the multiplicative semigroup of nonzero elements of $R$. We define for every ordinal $\alpha$ a subsemigroup $S_{\alpha}$ of $R^{*}$ as follows:

$S_{0}$ is the group of units of $R$.

$S_{\alpha}=\cup S_{\beta}, \beta<\alpha$, for $\alpha$ a limit ordinal.

If $\beta=\alpha-1$ exists we say an element $a$ in $R^{*}$ with $a$ not in $S_{\beta}$ is an $\alpha-$ atom if $a R$ is maximal among the principal right ideals $b R$ with $b$ not in $S_{\beta} . S_{\alpha}$ is then defined as the subsemigroup of $R^{*}$ generated by $S_{\beta}$ and the set of $\alpha$-atoms.

Under the above assumption there exists an ordinal $\alpha_{0}$ minimal with the property that $R^{*}=S_{\alpha_{0}}$. We refer to $\alpha$-atoms for any $\alpha$ as generalized atoms. The next result follows immediately.

LemMA 1. Let $R$ be an integral domain with right $\mathrm{ACC}_{1}$. Then every nonzero non-unit $a$ in $R$ can be written as a product of generalized atoms.

Let $R$ be an integral domain with modular factor lattice and right $\mathrm{ACC}_{1}$. It then follows that the intersection of any two principal right ideals $a R, b R$ of $R$ is again a principal right ideal: $a R \cap b R=v R$. Further, any two elements $a, b$ in $R$ with $a R \cap b R \neq 0$ have a greatest common left divisor $d$ with $a R \sqcup b R=d R$. We write $[a, b]=v=a b^{\prime}$ if $a R \cap b R=v R \neq 0, a^{-1}[a, b]$ for $b^{\prime}$ and $(a, b)=d$ if $a R \sqcup b R=d R$. We write $[a R, b R]$ for the sublattice $\{c R ; a R \leq c R \leq b R\}$ of $V(a)$ if $a R \leq b R$. We need the following definition.

Definition. An element $a^{\prime}$ is related to an element $a$ through $d$ if there exists an element $b$ with $(a, b)=d$ and $b^{-1}[a, b]=a^{\prime}$.

In case $a R+b R=a R \sqcup b R=d R$ holds, it is clear that $a^{\prime}$ related to $a$ through $d$ 
implies that $a^{\prime}$ is similar to $a_{1}$ with $a=d a_{1}$. We recall that two elements $r, r^{\prime}$ in $R$ are called similar if $R / r R$ and $R / r^{\prime} R$ are isomorphic as $R$-modules (see [4] for details).

LEMMA 2. Let $b, a=a_{1} a_{2}$ be nonzero elements in an integral domain $R$ with right $\mathrm{ACC}_{1}$ and modular factor lattice. Then $b^{-1}\left[b, a_{1} a_{2}\right]=a_{1}^{\prime} a_{2}^{\prime}$ where $a_{1}^{\prime}$ is related to $a_{1}$ through $d_{1}$ and $a_{2}^{\prime}$ is related to $a_{2}$ through $d_{2}$ with $d_{1}$ a left factor of $d=(b, a)$ and $d_{2}$ related to $d$ through $d_{3}$ with $d_{3} R=a_{1} R \sqcup d R$.

Proof. We have $\left[b, a_{1} a_{2}\right]=\left[b, a_{1}\right] r$ for some $r$ in $R$; $\left[a_{1}^{-1}\left[b, a_{1}\right], a_{2}\right]=a_{1}^{-1}\left[b, a_{1}\right] r$ follows. This leads to $r=c^{-1}\left[c, a_{2}\right]$ with $c=a_{1}^{-1}\left[b, a_{1}\right]$ and the desired equation with $a_{1}^{\prime}=b^{-1}\left[b, a_{1}\right]$ and $a_{2}^{\prime}=c^{-1}\left[c, a_{2}\right]$. We see that $\left(b, a_{1}\right)=d_{1}$ is a left factor of $d$. It remains to consider $c R \sqcup a_{2} R=d_{2} R$. This leads to $a_{1} d_{2} R=\left(b R \cap a_{1} R\right) \sqcup a R=a_{1} R \cap(a R \sqcup b R)=$ $a_{1} R \cap d R$, the claimed relation.

We observe that the lattices $V\left(d_{1}\right), V\left(d_{2}\right)$ are isomorphic to lattices $V\left(t_{1}\right), V\left(t_{2}\right)$ respectively where $t_{1}$ and $t_{2}$ are factors of $d$. The statement is obvious for $V\left(d_{1}\right)$, since we can take $t_{1}=d_{1}$. To see the second part assume $d=d_{3} d_{4}$ for some element $d_{4}$. It follows that the lattices

$$
V\left(d_{4}\right)=\left[d_{4} R, R\right] \cong\left[d R, d_{3} R\right] \cong\left[a_{1} d_{2} R, a_{1} R\right] \cong\left[d_{2} R, R\right]=V\left(d_{2}\right)
$$

are isomorphic, since $V\left(a_{1} d_{2}\right)$ is modular and $d_{3} R=a_{1} R \sqcup d R, a_{1} d_{2} R=a_{1} R \cap d R$ holds.

COROLlaRY 1. Let $r=a_{1} \ldots a_{n}=b_{1} \ldots b_{m}$ be two factorizations of an element $r$. Then either $a_{1}$ is a left factor of $b_{1}$ or there exists an index $j(2 \leq j \leq n)$ and an element $c$ in $R$ such that

$$
r=a_{1} b_{1}^{\prime} \ldots b_{j-1}^{\prime} c b_{j+1} \ldots b_{m}
$$

and $a_{1}^{\prime} c=b_{j}$. The element $a_{1}^{\prime}$ is related to $a_{1}$ through $d_{0}$ and $b_{i}^{\prime}$ is related to $b_{i}$ through $d_{i}$ for $i=1, \ldots, j-1$. The lattice $V\left(d_{i}\right)$ is isomorphic to $V\left(t_{i}\right)$ for $i=0, \ldots, j-1$ where $t_{i}$ is a factor of $d$ defined as $d=\left(a_{1}, b_{1} \ldots b_{j-1}\right)$ with $d R \supset a_{1} R$.

For a proof it is only necessary to choose $j$ such that $a_{1}$ is not a left factor of $b_{1} \ldots b_{j-1}$, but a left factor of $b_{1} \ldots b_{j}$ which then will equal to $\left[a_{1}, b_{1} \ldots b_{j-1}\right] c$ for some $c$ in $R$, defining the $c$ above. The rest follows from Lemma 2 .

Lemma 3. Let $R$ be an integral domain with right $\mathrm{ACC}_{1}$ and modular factor lattice. Assume $R=S_{\alpha_{0}}$ and that $\beta$ is an ordinal with $0 \leq \beta<\alpha_{0}, \gamma=\beta+1$. Then

(i) $a b$ is in $S_{\beta}$ if and only if $a, b$ are in $S_{\beta}$ for elements $a, b$ in $R$.

(ii) If $x$ is a $\gamma$-atom, $x=d y$ for $d$ in $S_{\beta}, y$ in $R$ then $y$ is a $\gamma$-atom.

(iii) If $a=n_{1} x_{1} n_{2} x_{2} \ldots n_{k} x_{k} n_{k+1}=m_{1} y_{1} m_{2} y_{2} \ldots m_{t} y_{i}$ is an element in $S_{\gamma}$ with $x_{i}, y_{j}$ $\gamma$-atoms and $n_{i}, m_{j}$ elements in $S_{\beta}$ for all $i, j$, and $r$ is in $R$ then $t \leq k$.

Proof. We prove these statements simultaneously by transfinite induction on $\beta$. Let $\beta=0$. Then (i) is obvious, (ii) is true since $d$ will be a unit and $y=d^{-1} x$ is a 1 -atom if and only if $x$ is. Finally, (iii) follows from the Jordan-Hölder Theorem for modular lattices.

We now assume that the statements are true for all $\alpha<\beta, \beta$ a non-limit ordinal. (The arguments in case $\beta$ is a limit ordinal are only slightly different and will be omitted). 
To prove ( $i$ ) let $r$ be an element in $R$, not contained in $S_{\beta}$. Given any natural number $q$, there exist $\beta$-atoms $x_{1}, \ldots, x_{q}$ with $r=x_{1} \ldots x_{q} r_{q}$ for some element $r_{q}$ in $R$. This together with (iii) applied to $S_{\beta}$ proves (i).

(ii) Let $x$ be a $\gamma$-atom, $d$ in $S_{\beta}, x=d y$. The element $y$ is not contained in $S_{\beta}, y=z r$ for some $\gamma$-atom $z$ with some $r$ in $R$ and $x=d z r$ follows. Using (i) we know that $d z$ is not in $S_{\beta}$, and since $x$ is a $\gamma$-atom, we conclude that $r$ must be a unit in $R$ and $y$ is a $\gamma$-atom.

(iii) We observe that $x^{\prime}$ is a $\gamma$-atom if $x^{\prime}$ is related to a $y$-atom $x$ through $d$ with $d R \supset x R$. This is obvious if we apply (ii) with $x=d y$ to obtain that $y$ is a $\gamma$-atom and recall that $V\left(x^{\prime}\right)$ and $V(y)$ are isomorphic lattices. Similarly, if $a^{\prime}$ is related to $a$ through $d$ with $a$ in $S_{\beta}$ it follows that $a^{\prime}$ is in $S_{\beta}$.

If we apply Corollary 1 to the two factorizations

$$
a=n_{1} x_{1} n_{2} \ldots n_{k} x_{k} n_{k+1}=m_{1} y_{1} \ldots m_{t} y_{t} r
$$

we see that we can assume that $n_{1}=1$. A second application of this Corollary shows that either $x_{1}$ is a left factor of $m_{1}$ or, in case $m_{1}$ equals 1 , of $y_{1}$, or

$$
a=x_{1} n_{2} \ldots n_{k} x_{k} n_{k+1}=x_{1} m_{1}^{\prime} y_{1}^{\prime} \ldots m_{j}^{\prime} c_{1} m_{j+1} y_{j+1} \ldots r
$$

for some $j \leq t$, or $a=x_{1} m_{1}^{\prime} y_{1}^{\prime} \ldots m_{i}^{\prime} y_{i}^{\prime} c_{2}$. The elements $m_{i}^{\prime}$ are still in $S_{\beta}$, the $y_{i}^{\prime}$ are still $\gamma$-atoms, $x_{1}^{\prime} c_{1}=y_{j}$ implies $c_{1}$ is a unit in $R$ and $x_{1}^{\prime} c_{2}=r$ holds in the second case for some element $c_{2}$ in $R$.

The statement (iii) follows by induction on $k$ after cancelling $x_{1}$.

COROLlaRY 2. Let $x$ be an $\alpha$-atom, y a $\beta$-atom with $\alpha<\beta$ in a ring $R$ satisfying the assumptions of Lemma 3. Then $x y=y^{\prime}$ or $x y=y^{\prime} x^{\prime}$ for a $\beta$-atom $y^{\prime}$ and an $\alpha$-atom $x^{\prime}$.

Proof. We have $x y=\bar{y} r$ for some $\beta$-atom $\bar{y}$ and an element $r$ in $R$. If $\bar{y}=x y_{1}$ for some element $y_{1}$ in $R$ we obtain that $y_{1}$ is a $\beta$-atom, $y=y_{1} r$ and $r$ must be a unit; $x y=\bar{y} r=y^{\prime}$ with $y^{\prime}$ a $\beta$-atom. If $\bar{y}$ is not contained in $x R$ we have $x R \cap \bar{y} R=x y_{1} R=\bar{y} x_{1} R$ for elements $y_{1}$ and $x_{1}$ in $R$. This leads to $x y_{1} c=\bar{y} x_{1} c=x y=\bar{y} r$ for some element $c$ in $R$. Since $y_{1}$ is a $\beta$-atom, $c$ must be a unit in $R$. The element $x_{1}$ is an $\alpha$-atom and $x y=y^{\prime} x^{\prime}$ follows with $y^{\prime}=\bar{y}, x^{\prime}=x_{1} c$.

THEOREM 1 . Let $R$ be an integral domain with modular factor lattice and right $A_{C C}$. Then every nonzero non-unit $a$ in $R$ can be written as

$$
a=x_{1}^{\left(\alpha_{1}\right)} \ldots x_{n_{1}}^{\left(\alpha_{1}\right)} x_{1}^{\left(\alpha_{2}\right)} \ldots x_{n_{2}}^{\left(\alpha_{2}\right)} \ldots x_{1}^{\left(\alpha_{k}\right)} \ldots x_{n_{k}}^{\left(\alpha_{k}\right)}
$$

where the $x_{i}^{\left(\alpha_{i}\right)}$ are $\alpha_{i}$-atoms in $R$ with $\alpha_{1}>\alpha_{2}>\ldots>\alpha_{k}$. If

$$
a=y_{1}^{\left(\beta_{1}\right)} \ldots y_{m_{1}}^{\left(\beta_{1}\right)} y_{1}^{\left(\beta_{2}\right)} \ldots y_{m_{2}}^{\left(\beta_{2}\right)} \ldots y_{1}^{\left(\beta_{1}\right)} \ldots y_{m_{1}}^{\left(\beta_{1}\right)}
$$

is another such factorization with $\beta_{j}$-atoms $y_{i}^{\left(\beta_{i}\right)}$ and $\beta_{1}>\beta_{2}>\ldots>\beta_{1}$ then we have $t=k$, $n_{i}=m_{i}, \alpha_{i}=\beta_{i}$ for $i=1, \ldots, k$ and there exist units $\varepsilon_{i}(i=0, \ldots, k)$ with $\varepsilon_{0}=\varepsilon_{k}=1$ and

$$
\varepsilon_{i-1}^{-1} y_{1}^{\left(\alpha_{1}\right)} \ldots y_{n_{1}}^{\left(\alpha_{1}\right)} \varepsilon_{i}=x_{1}^{\left(\alpha_{i}\right)} \ldots x_{n_{1}}^{\left(\alpha_{1}\right)}
$$

for $i=1, \ldots, k$. 
Proof. It follows from Lemma 1 and Corollary 2 that a factorization $\left({ }^{*}\right)$ exists for every nonzero non-unit $a$ in $R$. The ordinal $\alpha_{1}$ is determined by $a$ as the ordinal $\alpha$ minimal with the property that $a$ is contained in $S_{\alpha}$. We obtain $\alpha_{1}=\beta_{1}$ and $n_{1}=m_{1}$ using (iii) in Lemma 3. That $x_{1}^{\left(\alpha_{1}\right)} \ldots x_{n}^{\left(\alpha_{1}\right)}=y_{1}^{\left(\beta_{1}\right)} \ldots y_{n}^{\left(\beta_{1}\right)} \varepsilon_{1}$ for some unit $\varepsilon_{1}$ in $R$ follows from a repeated application of Corollary 1 . We have to observe that a $\beta$-atom cannot have a factor which is related to an $\alpha$-atom through $d$ for $d$ in $S_{\gamma}$ and $\gamma<\alpha, \beta<\alpha$. An easy induction finishes the proof of the theorem.

The above results can be applied to weak Bezout domains with right $\mathrm{ACC}_{1}$. We recall that an integral domain in which the sum and the intersection of any two principal right ideals is principal whenever the intersection is nonzero is called a weak Bezout domain. We make the following definition. Let $R$ be a weak Bezout domain with right $\mathrm{ACC}_{1}$. Two $\beta$-atoms $x$ and $y$ are called linked if there exist $\beta$-atoms $x=z_{0}, z_{1}, z_{2}, \ldots, z_{n}=y$ in $R$ such that either $z_{i}$ is similar to $z_{i+1}$ or that $z_{i}=d_{i} z_{i+1}$ or that $d_{i} z_{i}=z_{i+1}$ for $d_{i}$ in $S_{\alpha}$ with $\alpha<\beta$ for $i=0, \ldots, n-1$. With this notation we formulate the following addition to Theorem 1.

Corollary. Let $R$ be a weak Bezout domain with right $\mathrm{ACC}_{1}$. Let $a=x_{1} \ldots x_{n}=$ $y_{1} \ldots y_{n}$ be two factorizations of an element $a$ in $R$ into $\beta$-atoms $x_{i}, y_{j}$ respectively. Then there exists a permutation $\sigma$ of $\{1, \ldots, n\}$ such that $x_{i}$ and $y_{\sigma(i)}$ are linked for $i=1, \ldots, n$.

The best possible result is obtained for local weak Bezout domains. Here we say a ring $R$ is local if the non-units form an ideal in $R$.

THEOREM 2. Let $R$ be a local weak Bezout domain with right $\mathrm{ACC}_{1}$.

(i) Let $a^{\prime}=x_{1}^{\left(\alpha_{1}\right)} x_{2}^{\left(\alpha_{2}\right)} \ldots x_{n}^{\left(\alpha_{n}\right)}=y_{1}^{\left(\beta_{1}\right)} \ldots y_{m}^{\left(\beta_{m}\right)}$ be two factorizations of an element $a$ in $R$ into $\alpha_{i}$-atoms $x_{i}^{\left(\alpha_{i}\right)}$ and $\beta_{j}$-atoms $y_{j}^{\left(\beta_{i}\right)}$, respectively, with $\alpha_{1} \geq \alpha_{2} \geq \ldots \geq \alpha_{n}$ and $\beta_{1} \geq \beta_{2} \geq$ $\ldots \geq \beta_{m}$. Then $n=m, \alpha_{i}=\beta_{i}$ and there exist units $\varepsilon_{i}(i=0, \ldots, n)$ with $1=\varepsilon_{0}=\varepsilon_{n}$ and $\dot{x}_{i}^{\left(\alpha_{i}\right)}=\varepsilon_{i=1}^{-1} y_{i}^{\left(\alpha_{i}\right)} \varepsilon_{i}$.

(ii) Let $x$ be an $\alpha$-atom, y a $\beta$-atom in $R$ with $\alpha<\beta$. Then $x y$ is a $\beta$-atom $y^{\prime}$ in $R$.

Proof. (i) We know that $n=m$ and $\alpha_{i}=\beta_{i}$ from Theorem 1. Assume $x$ and $y$ are $\alpha$ atoms with $x R \cap y R \neq 0$. It follows that $x R+y R=d R$ for some $d$ in $R$, and $x_{1} R+y_{1} R=$ $R$ where $x_{1}, y_{1}$ are defined through $x=d x_{1}, y=d y_{1}$. If $d R \neq x R$ and $d R \neq y R$ we see that $x_{1}$ and $y_{1}$ are still $\alpha$-atoms and a contradiction would be reached. This leaves us with $x R=y R=d R$. This comment applied to $x_{1}^{\left(\alpha_{1}\right)}$ and $y_{1}^{\left(\alpha_{1}\right)}$ leads to $x_{1}^{\left(\alpha_{1}\right)}=y_{1}^{\left(\alpha_{1}\right)} \varepsilon_{1}$ for some unit $\varepsilon_{1}$ in $R$. Cancellation of $x_{1}^{\left(\alpha_{1}\right)}$ and induction after $n$ ends the proof of $(i)$. To prove (ii) let $x$ be an $\alpha$-atom, $y$ a $\beta$-atom with $\alpha<\beta$. We have $x y=\bar{y} r$ for some $\beta$-atom $\bar{y}$ in $R$ (Corollary 2) and as in the argument above $x R+\bar{y} R=x R$ must follow since $R$ is a local weak Bezout domain. This implies that only the first case of the two cases treated in the proof of Corollary 2 can happen and proves the result.

We conclude with a few examples.

(1) The first example shows that there exist integral domains $R$ with right $\mathrm{ACC}_{1}$ such that $V(a)=\{b R ; b R \geq a R\}$ is a lattice for every $a \neq 0$ in $R$, but $R$ does not have modular factor lattice. Let $K$ be any commutative field, $K[t]_{(t)}=A$ the localization of the polynomial ring $K[t]$ on the prime ideal $(t)$. There exists a monomorphism $\sigma$ from $A$ into 
$A$ which maps $t$ to $t^{2}$ and fixes the elements in $K$. Finally let $R_{1}=A[[x, \sigma]]$ be the skew power series ring in one variable $x$ with the elements $\sum_{0}^{\infty} a_{i} x^{i}, a_{i}$ in $A$, and $x a=\sigma(a) . x$. We know from [3] that $R_{1}$ is an integral domain with right and left $\mathrm{ACC}_{1}$, the intersection of any two principal right ideals is again a principal right ideal and the numbers of irreducible factors in a factorization of $a$ is bounded by some constant dependent on $a$. This implies that $V(a)$ is a lattice, but this lattice is not modular in general as can be seen for $a=x t$ for example.

(2) One can choose the field $K$ in the first example so that there exists a monomorphism $\tau$ from $A$ into $K$. Let $R_{2}$ be the free power series ring $R_{2}=A\{\{X, \tau\}\}$ in a set on non-commuting variables $X=\left\{x_{i}\right\}, i \in I$, such that $A$ is not in the center, but that $a . x_{i}=x_{i} \tau(a)$ determines the multiplication. $R_{2}$ is a local Bezout domain with right $\mathrm{ACC}_{1}$ and $R_{2}^{*}=S_{2}$.

(3) The last example shows that the uniqueness statement in Theorem 1 can be correct for elements in $S_{\alpha}$ in an integral domain $R$ with right $\mathrm{ACC}_{1}$, but wrong for elements in $S_{\beta}$ with $\beta>\alpha$. We use the local ring $A$ with its monomorphism $\tau$ from Example 2. Let $T$ be the skew power series ring $A[[x, \tau]]$ in one variable over $A$ with elements $\sum_{i=0}^{\infty} x^{i} a_{i}, a_{i} \in A$ and $a x=x \tau(a)$. Let $R_{3}$ be the subring of $T$ consisting of all those elements for which the coefficient $a_{1}$ of $x$ is zero. The 1 -atoms are of the form $t \varepsilon$ for $\varepsilon$ a unit in $R_{3}$ and every element in $S_{1}$ can be factored uniquely up to units. But $x^{2}$ and $x^{3}$ are both 2-atoms and $x^{6}=\left(x^{2}\right)^{3}=\left(x^{3}\right)^{2}$.

\section{REFERENCES}

1. R. A. Beauregard, Infinite primes and unique factorization in a principal right ideal domain, Trans. Amer. Math. Soc. 141 (1969), 245-253.

2. H. H. Brungs, Generalized discrete valuation rings, Canad. J. Math. 21 (1969), 1404-1408. 45-66.

3. H. H. Brungs, Ringe mit eindeutiger Faktorzerlegung, J. Reine Angew. Math. 236 (1969),

4. P. M. Cohn, Free rings and their relations (Academic Press, 1971).

5. A. Jategaonkar, Left principal ideal rings, Lecture Notes in Mathematics 123 (SpringerVerlag, 1970).

Department of Mathematics

UNIVERSITY OF AlBERTA

EDMONTON

Alberta

CANADA 